Korean J. Math. 20 (2012), No. 3, pp. 343-352

\title{
PROJECTIVE REPRESENTATIONS OF A QUIVER WITH THREE VERTICES AND TWO EDGES AS $R[x]$-MODULES
}

\author{
JUNCHEOL HAN AND SANGWON PARK*
}

\begin{abstract}
In this paper we show that the projective properties of representations of a quiver $Q=\bullet \rightarrow \bullet \rightarrow \bullet$ as left $R[x]$-modules. We show that if $P$ is a projective left $R$-module then $0 \longrightarrow 0 \longrightarrow P[x]$ is a projective representation of a quiver $Q$ as $R[x]$-modules, but $P[x] \longrightarrow 0 \longrightarrow 0$ is not a projective representation of a quiver $Q$ as $R[x]$-modules, if $P \neq 0$. And we show a representation $0 \longrightarrow P[x] \stackrel{i d}{\longrightarrow} P[x]$ of a quiver $Q$ is a projective representation, if $P$ is a projective left $R$-module, but $P[x] \stackrel{i d}{\longrightarrow} P[x] \longrightarrow 0$ is not a projective representation of a quiver $Q$ as $R[x]$-modules, if $P \neq$ 0 . Then we show a representation $P[x] \stackrel{i d}{\longrightarrow} P[x] \stackrel{i d}{\longrightarrow} P[x]$ of a quiver $Q$ is a projective representation, if $P$ is a projective left $R$ module.
\end{abstract}

\section{Introduction}

A quiver is just a directed graph with vertices and edges (arrows) [1]. We may consider many different types of quivers. We allow multiple edges and multiple arrows, and edges going from a vertex back to the same vertex. Originally a representation of the quiver assigned a vector space to each vertex - and a linear map to each edge (or arrow) - with the linear map going from the vector space assigned to the initial vertex to

Received August 8, 2012. Revised September 2, 2012. Accepted September 5, 2012 .

2010 Mathematics Subject Classification: 16E30, 13C11, 16D80.

Key words and phrases: quiver, projective module, injective module, projective representation, injective representation.

This study was supported by research funds from Dong-A University.

*Corresponding author. 
the one assigned to the terminal vertex. For example, a representation of the quiver $Q=\bullet \rightarrow \bullet \rightarrow \bullet$ is $V_{1} \stackrel{f}{\longrightarrow} V_{2} \stackrel{g}{\longrightarrow} V_{3}, V_{1}, V_{2}$ and $V_{3}$ are vector spaces and $f, g$ are linear maps (morphisms). Then we extend this representation to the left $R$-modules, a representation of the quiver $Q=\bullet \rightarrow \bullet \rightarrow \bullet$ is $M_{1} \stackrel{\alpha}{\longrightarrow} M_{2} \stackrel{\beta}{\longrightarrow} M_{3}, M_{1}, M_{2}$ and $M_{3}$ are left $R$ modules and $\alpha, \beta$ are $R$-linear maps. Throughout this paper a (the) quiver $Q$ means a (the) quiver $Q=\bullet \rightarrow \bullet \rightarrow \bullet$.

If $M$ is a left $R$-module, then the polynomial $M[x]$ is a left $R[x]$ module defined by

$$
\begin{aligned}
& r\left(m_{0}+m_{1} x+m_{2} x^{2} \cdots+m_{i} x^{i}\right)=r m_{0}+r m_{1} x+r m_{2} x^{2}+\cdots+r m_{i} x^{i} \\
& x\left(m_{0}+m_{1} x+m_{2} x^{2} \cdots+m_{i} x^{i}\right)=m_{0} x+m_{1} x^{2}+m_{2} x^{3}+\cdots+m_{i} x^{i+1} .
\end{aligned}
$$

We call $M[x]$ a polynomial module. Similarly we can define the power series $M[[x]]$ as a left $R[x]$-module and we call a power series modules.

The following diagram

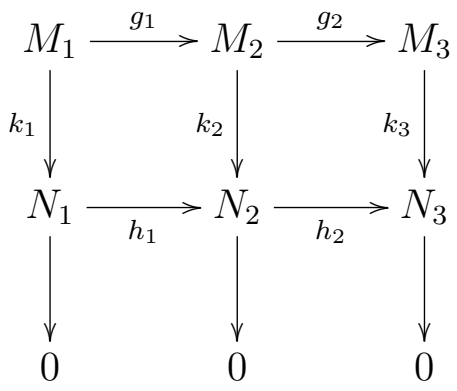

is commutative which means $h_{1} k_{1}=k_{2} g_{1}, h_{2} k_{2}=k_{3} g_{2}$, and $k_{3} g_{2} g_{1}=$ $h_{2} h_{1} k_{1}=h_{2} k_{2} g_{1}$.

In [3] a homotopy of a quiver was developed and in [2] a cyclic quiver ring was studies. The theory of projective representations were developed in [4] and the theory of injective representations were studied in [5]. Recently, in [7] injective covers and envelopes of representations of linear quivers were studied, and in [6] properties of multiple edges of quivers were studied.

Definition 1.1. [9] A left $R$-module $P$ is said to be projective if given any surjective linear map $\sigma: M^{\prime} \rightarrow M$ and any linear map $h: P \rightarrow M$ 
, there is a linear map $g: P \rightarrow M^{\prime}$ such that $\sigma \circ g=h$. That is

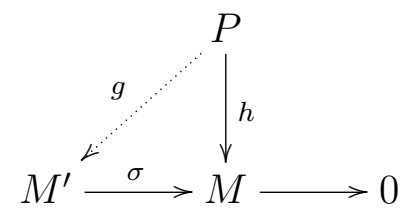

can always be completed to a commutative diagram.

Definition 1.2. Let $M_{1}, M_{2}, M_{3}, N_{1}, N_{2}, N_{3}, P_{1}, P_{2}, P_{3}$ be left $R$-modules, and $k_{1}: M_{1} \rightarrow N_{1}, k_{2}: M_{2} \rightarrow N_{2}, k_{3}: M_{3} \rightarrow N_{3}$ be onto $R$-linear maps. A representation $P_{1} \stackrel{f_{1}}{\longrightarrow} P_{2} \stackrel{f_{2}}{\longrightarrow} P_{3}$ of a quiver $Q=\bullet \rightarrow \bullet \rightarrow \bullet$ is called a projective representation if every diagram of representations

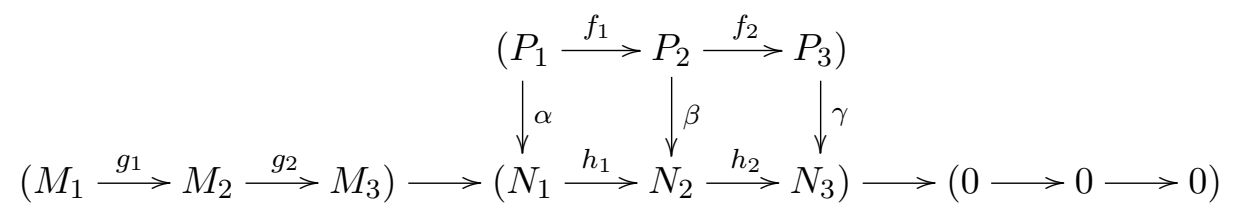

with

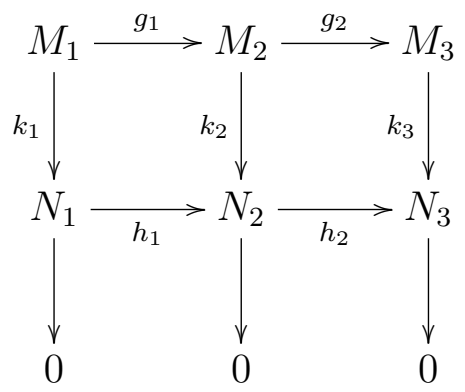

can be completed to a commutative diagram as follows :

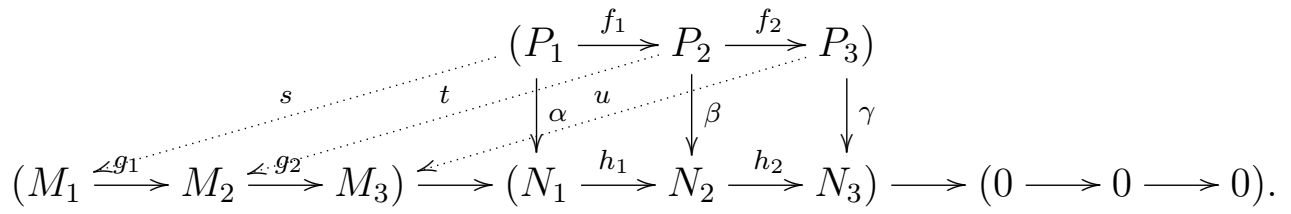




\section{Projective representation of a quiver $Q$ as $R[x]$-modules}

Theorem 2.1. If $P$ is a projective left $R$-module then $0 \longrightarrow 0 \longrightarrow P[x]$ is a projective representation of a quiver $Q$ as $R[x]$-modules.

Proof. Let $M_{1}, M_{2}, M_{3}, N_{1}, N_{2}, N_{3}$ be left $R[x]$-modules, and $k_{1}: M_{1} \rightarrow$ $N_{1}, k_{2}: M_{2} \rightarrow N_{2}, k_{3}: M_{3} \rightarrow N_{3}$ be onto $R[x]$-linear maps, and $f: P[x] \rightarrow N_{3}$ be an $R[x]$-linear map. And consider the following diagrams

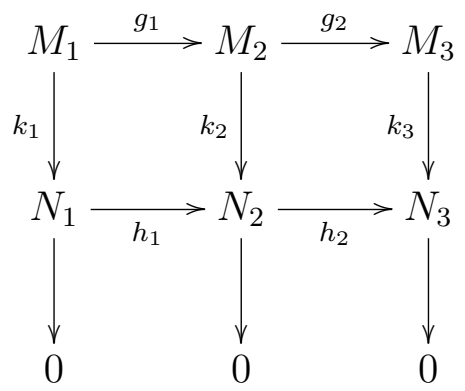

and

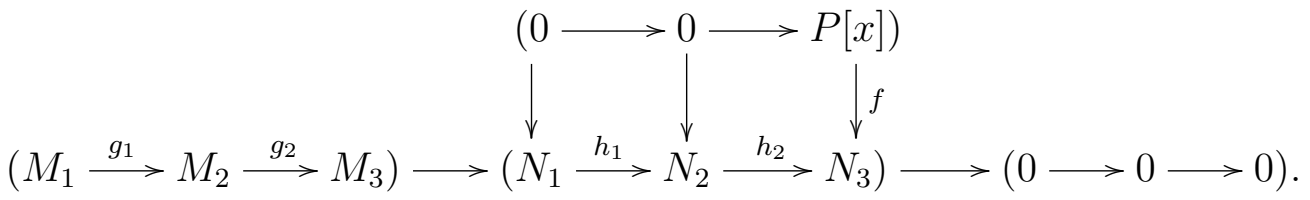

Since $P$ is a projective left $R$-module, there exists an $R$-linear map $t$ : $P \rightarrow M_{3}$ such that $k_{3} t=\left.f\right|_{P}$. Define $\bar{t}: P[x] \rightarrow M_{3}$ by $\bar{t}\left(p_{0}+p_{1} x+\right.$ $\left.\cdots+p_{n} x^{n}\right)=t\left(p_{0}\right)+t\left(p_{1}\right) x+\cdots+t\left(p_{n}\right) x^{n}$. Then

$$
\begin{aligned}
& k_{3} \bar{t}\left(p_{0}+p_{1} x+\cdots+p_{n} x^{n}\right) \\
& =k_{3}\left(t\left(p_{0}\right)+t\left(p_{1}\right) x+\cdots+t\left(p_{n}\right) x^{n}\right) \\
& =\left(k_{3} t\right)\left(p_{0}\right)+\left(k_{3} t\right)\left(p_{1}\right) x+\cdots+\left(k_{3} t\right)\left(p_{n}\right) x^{n} \\
& =\left.f\right|_{P}\left(p_{0}\right)+\left.f\right|_{P}\left(p_{1}\right) x+\cdots+\left.f\right|_{P}\left(p_{n}\right) x^{n} \\
& =f\left(p_{0}+p_{1} x+\cdots+p_{n} x^{n}\right) .
\end{aligned}
$$

So we have $k_{3} \bar{t}=f$. Therefore, we can complete the following diagram 


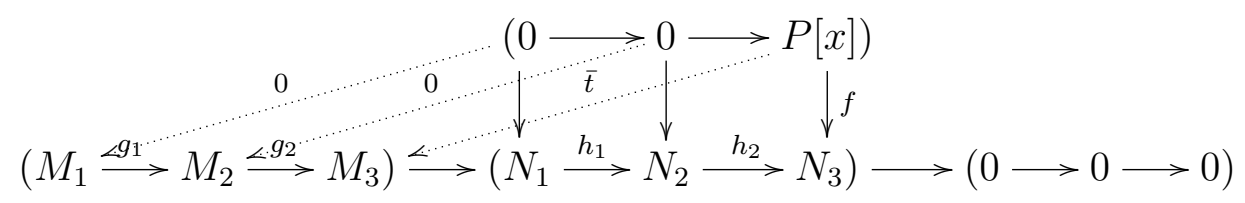

as a commutative diagram. Hence, $0 \rightarrow 0 \rightarrow P[x]$ is a projective representation of a quiver $Q$ as $R[x]$-modules.

REMARK 2.2. We see that $P[x] \longrightarrow 0 \longrightarrow 0$ is not a projective representation of a quiver $Q$ as $R[x]$-modules if $P \neq 0$, because the following diagram

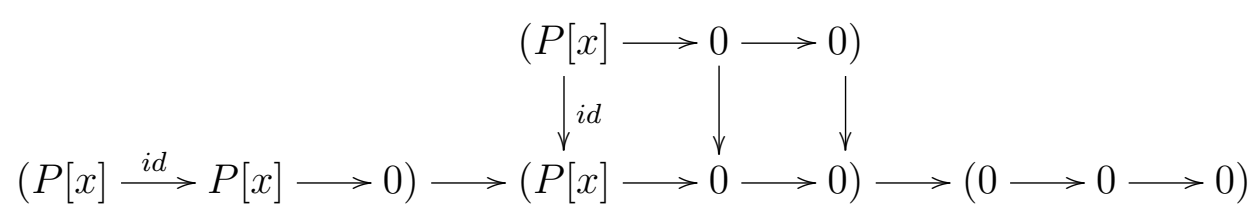

can not be completed as a commutative diagram with

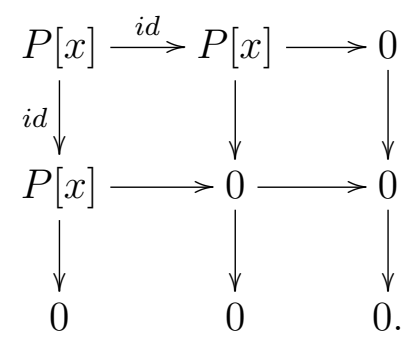

Similarly, $P[[x]] \longrightarrow 0 \longrightarrow 0$ is not a projective representation of a quiver $Q$ as $R[x]$-modules if $P \neq 0$.

Corollary 2.3. If $P$ is a projective left $R$-module then $0 \longrightarrow 0 \longrightarrow P[[x]]$ is a projective representation of a quiver $Q$ as $R[x]$ modules.

Let $Z_{n}$ denotes the ring of integers modulo $n$.

ExAmple 2.4. Let $R=Z_{6}$, then $P=Z_{2}$ is a projective $Z_{6}$-module. $0 \longrightarrow 0 \longrightarrow Z_{2}[x]$ is a projective representation of a quiver $Q$ as $Z_{6}[x]$ modules. 
TheOREM 2.5. A representation $0 \longrightarrow P[x] \stackrel{i d}{\longrightarrow} P[x]$ of a quiver $Q$ is a projective representation, if $P$ is a projective left $R$-module.

Proof. Let $f: P[x] \longrightarrow N_{2}$ be an $R[x]$-linear map and $k_{1}: M_{1} \longrightarrow$ $N, k_{2}: M_{2} \longrightarrow N_{2} k_{3}: M_{3} \longrightarrow N_{3}$ be onto $R[x]$-linear maps and choose $h_{2} f: P[x] \longrightarrow N_{3}$ as an $R[x]$-linear map. Consider the following diagrams

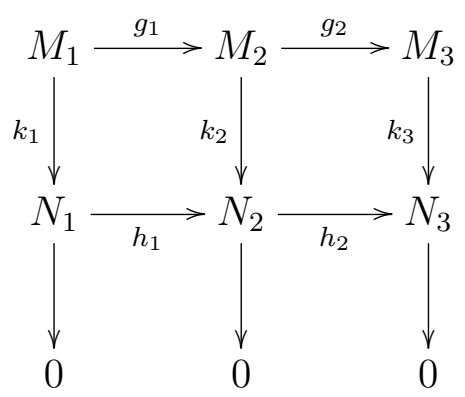

and

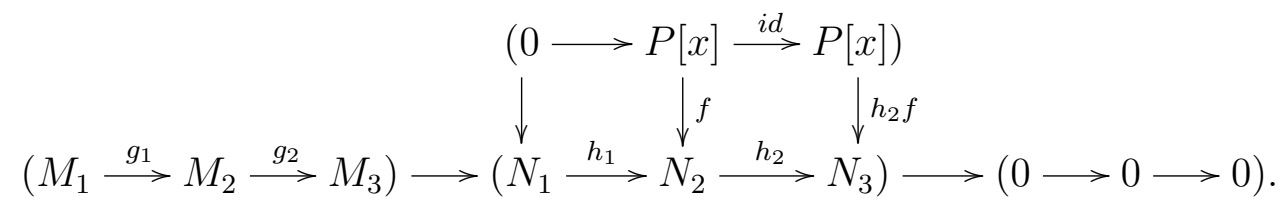

Then since $P$ is a projective left $R$-module, there exists $t: P \rightarrow M_{2}$ such that $k_{2} t=\left.f\right|_{P}$.

Define $\bar{t}: P[x] \rightarrow M_{2}$ by $\bar{t}\left(n_{0}+n_{1} x+\cdots+n_{i} x^{i}\right)=t\left(n_{0}\right)+t\left(n_{1}\right) x+\cdots+$ $t\left(n_{i}\right) x^{i}$. Then

$$
\begin{aligned}
& k_{2} \bar{t}\left(n_{0}+n_{1} x+\cdots+n_{i} x^{i}\right) \\
& =k_{2}\left(t\left(n_{0}\right)+t\left(n_{1}\right) x+\cdots+t\left(n_{i}\right) x^{i}\right) \\
& =\left(k_{2} t\right)\left(n_{0}\right)+\left(h_{2} t\right)\left(n_{1}\right) x+\cdots+\left(h_{2} t\right)\left(n_{i}\right) x^{i} \\
& =\left.f\right|_{P}\left(n_{0}\right)+\left.f\right|_{P}\left(n_{1}\right) x+\cdots+\left.f\right|_{P}\left(n_{i}\right) x^{i} \\
& =f\left(n_{0}+n_{1} x+\cdots+n_{i} x^{i}\right) .
\end{aligned}
$$

Now let $g_{2} \bar{t}: P[x] \rightarrow M_{3}$ be an $R[x]$-linear map, then $k_{3} g_{2} \bar{t}=h_{2} k_{2} \bar{t}=$ $h_{2} f$. So we have the following commutative diagram 


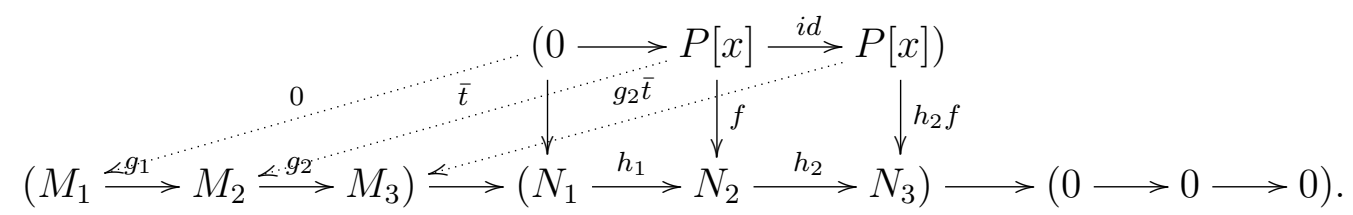

Hence, $P$ is a projective left $R$-module implies a representation $0 \longrightarrow P[x] \stackrel{i d}{\longrightarrow} P[x]$ is a projective representation of a quiver $Q$.

REMARK 2.6. We see that $P[x] \stackrel{i d}{\longrightarrow} P[x] \longrightarrow 0$ is not a projective representation of a quiver $Q$ as $R[x]$-modules if $P \neq 0$, because the following diagram

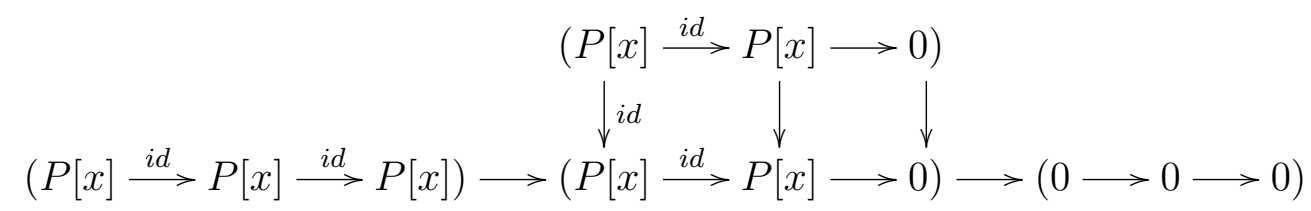

can not be completed as a commutative diagram with

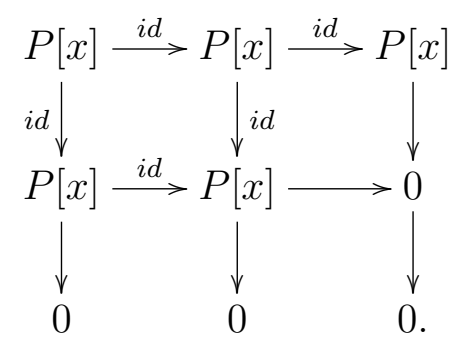

Similarly, $P[[x]] \stackrel{i d}{\longrightarrow} P[[x]] \longrightarrow 0$ is not a projective representation of a quiver $Q$ as $R[x]$-modules if $P \neq 0$.

Corollary 2.7. If $P$ is a projective left $R$-module then $0 \longrightarrow P[[x]] \stackrel{i d}{\longrightarrow} P[[x]]$ is a projective representation of a quiver $Q$ as $R[x]$-modules.

ExAmple 2.8. Let $R=Z_{6}$, then $P=Z_{2}$ is a projective $Z_{6}$-module. $0 \longrightarrow Z_{2}[x] \stackrel{i d}{\longrightarrow} Z_{2}[x]$ is a projective representation of a quiver $Q$ as $Z_{6}[x]$-modules. 
TheOREM 2.9. A representation $P[x] \stackrel{i d}{\longrightarrow} P[x] \stackrel{i d}{\longrightarrow} P[x]$ of a quiver $Q$ is a projective representation, if $P$ is a projective left $R$-module.

Proof. Let $f: P[x] \longrightarrow N_{1}$ be an $R[x]$-linear map and $k_{1}: M_{1} \longrightarrow N$, $k_{2}: M_{2} \longrightarrow N_{2} k_{3}: M_{3} \longrightarrow N_{3}$ be onto $R[x]$-linear maps and choose $h_{1} f: P[x] \longrightarrow N_{2}$ and $h_{2} h_{1} f: P[x] \longrightarrow N_{3}$ as $R[x]$-linear maps. And consider the following diagrams

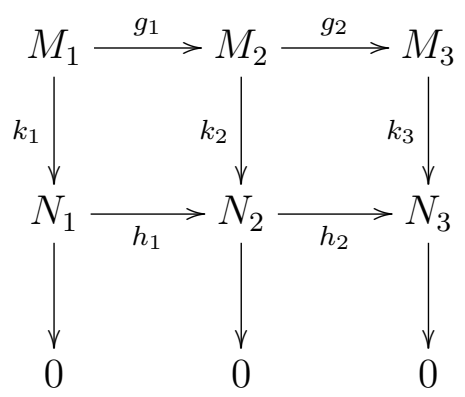

and

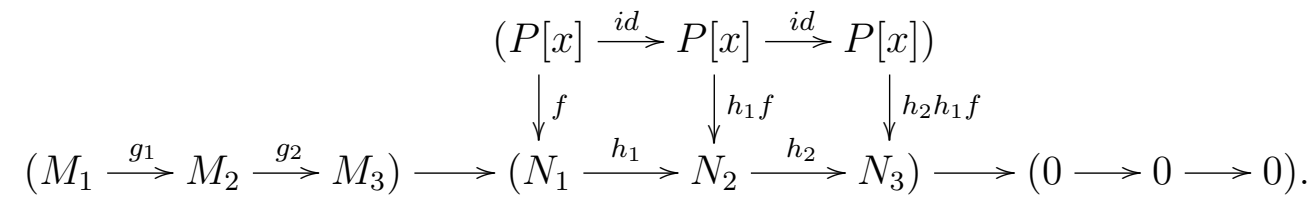

Then since $P$ is a projective left $R$-module, there exists $t: P \rightarrow M_{1}$ such that $k_{1} t=\left.f\right|_{P}$.

Define $\bar{t}: P[x] \rightarrow M_{1}$ by $\bar{t}\left(n_{0}+n_{1} x+\cdots+n_{i} x^{i}\right)=t\left(n_{0}\right)+t\left(n_{1}\right) x+\cdots+$ $t\left(n_{i}\right) x^{i}$. Then

$$
\begin{aligned}
& k_{1} \bar{t}\left(n_{0}+n_{1} x+\cdots+n_{i} x^{i}\right) \\
& =k_{1}\left(t\left(n_{0}\right)+t\left(n_{1}\right) x+\cdots+t\left(n_{i}\right) x^{i}\right) \\
& =\left(k_{1} t\right)\left(n_{0}\right)+\left(k_{1} t\right)\left(n_{1}\right) x+\cdots+\left(k_{1} t\right)\left(n_{i}\right) x^{i} \\
& =\left.f\right|_{P}\left(n_{0}\right)+\left.f\right|_{P}\left(n_{1}\right) x+\cdots+\left.f\right|_{P}\left(n_{i}\right) x^{i} \\
& =f\left(n_{0}+n_{1} x+\cdots+n_{i} x^{i}\right) .
\end{aligned}
$$

Now let $g_{1} \bar{t}: P[x] \rightarrow M_{2}$ be an $R[x]$-linear map, then $k_{2} g_{1} \bar{t}=h_{1} k_{1} \bar{t}=$ $h_{1} f$. And let $g_{2} g_{1} \bar{t}: P[x] \rightarrow M_{3}$ be an $R[x]$-linear map. Then $k_{3} g_{2} g_{1} \bar{t}=$ $h_{2} k_{2} g_{1} \bar{t}=h_{2} h_{1} k_{1} \bar{t}=h_{2} h_{1} f$. So we have the following commutative diagram 
$(P[x] \stackrel{i d}{\longrightarrow} P[x] \stackrel{i d}{\longrightarrow} P[x])$
$\left(M_{1} \stackrel{g_{1}-M_{2}}{\longrightarrow} M_{2} \stackrel{g_{2}}{\longrightarrow} M_{3}\right) \stackrel{g_{1} \bar{t}}{\longrightarrow}\left(N_{1} \stackrel{h_{1}}{\longrightarrow} N_{2} \stackrel{h_{2}}{\longrightarrow} N_{3}\right) \longrightarrow(0 \longrightarrow 0 \longrightarrow 0)$.

Hence, $P$ is a projective left $R$-module implies a representation $P[x] \stackrel{i d}{\longrightarrow} P[x] \stackrel{i d}{\longrightarrow} P[x]$ is a projective representation of a quiver $Q$.

Corollary 2.10. If $P$ is a projective left $R$-module then $P[[x]] \stackrel{i d}{\longrightarrow} P[[x]] \stackrel{i d}{\longrightarrow} P[[x]]$ is a projective representation of a quiver $Q$ as $R[x]$-modules.

ExAmPle 2.11. Let $R=Z_{6}$, then $P=Z_{2}$ is a projective $Z_{6}$-module. $Z_{2}[x] \stackrel{i d}{\longrightarrow} Z_{2}[x] \stackrel{i d}{\longrightarrow} Z_{2}[x]$ is a projective representation of a quiver $Q$ as $Z_{6}[x]$-modules.

\section{References}

[1] R. Diestel, Graph Theory, G.T.M. No.88, Springer-Verlag, New York (1997).

[2] E. Enochs, I. Herzog, S. Park, Cyclic quiver rings and polycyclic-by-finite group rings, Houston J. Math. (1), 25 (1999) 1-13.

[3] E. Enochs, I. Herzog, A homotopy of quiver morphism with applications to representations, Canad J. Math. (2), 51 (1999), 294-308.

[4] S. Park, Projective representations of quivers, IJMMS(2), 31 (2002), 97-101.

[5] S. Park, D. Shin, Injective representation of quiver, Commun. Korean Math. Soc. (1), 21 (2006), 37-43.

[6] S. Park, Injective and projective properties of representation of quivers with $n$ edges, Korean J. Math. (3), 16 (2008), 323-334.

[7] S. Park, E. Enochs, H. Kim Injective covers and envelopes of representation of linear quiver, Commun. Algebra (2), 37 (2009), 515-524.

[8] R.S. Pierce, Associative Algebras, G.T.M. No.173, Springer-Verlag, New York (1982).

[9] J. Rotman, An Introduction to Homological Algebra, Academic Press Inc., New York (1979).

Department of Mathematics Educations

Pusan National University

Pusan 609-735, Korea

E-mail: jchan@puan.ac.kr 
Department of Mathematics

Dong-A University

Pusan 604-714, Korea

E-mail: swpark@donga.ac.kr 